\title{
The Roles of Feature-Specific Task Set and Bottom-Up Salience in Attentional Capture: An ERP Study
}

\author{
Martin Eimer, Monika Kiss, Clare Press, and Disa Sauter \\ Birkbeck College, University of London
}

\begin{abstract}
We investigated the roles of top-down task set and bottom-up stimulus salience for feature-specific attentional capture. Spatially nonpredictive cues preceded search arrays that included a color-defined target. For target-color singleton cues, behavioral spatial cueing effects were accompanied by cueinduced N2pc components, indicative of attentional capture. These effects were only minimally attenuated for nonsingleton target-color cues, underlining the dominance of top-down task set over salience in attentional capture. Nontarget-color singleton cues triggered no N2pc, but instead an anterior N2 component indicative of top-down inhibition. In Experiment 2, inverted behavioral cueing effects of these cues were accompanied by a delayed N2pc to targets at cued locations, suggesting that perceptually salient but task-irrelevant visual events trigger location-specific inhibition mechanisms that can delay subsequent target selection.
\end{abstract}

Keywords: spatial attention, top-down control, vision, inhibition, event-related potentials

The distinction between endogenous, voluntary, and goaldirected attention shifts, and the exogenous, involuntary, and stimulus-driven capture of attention is central to theories of attentional processing. Endogenous attention refers to the selective processing of stimuli that is guided in a top-down fashion by current goals and intentions. In contrast, exogenous attention refers to the selection of objects that is driven in a bottom-up fashion by their perceptual salience, independently of their behavioral relevance as defined by current task sets.

Despite this clear-cut conceptual distinction, investigations of how observers' performance in visual search tasks is affected by top-down versus bottom-up factors have produced apparently inconsistent results. On the one hand, reaction times (RTs) during visual search for shape singletons (e.g., unique diamond targets presented among distractor circles) have been shown to be delayed by the presence of salient but task-irrelevant color singletons (e.g., Theeuwes, 1991), suggesting that such color singletons will capture attention in a bottom-up fashion, independently of current selection goals. On the other hand, studies by Folk and colleagues (Folk \& Remington, 1998; Folk, Remington, \& Johnston, 1992; Folk, Remington, \& Wright, 1994) have demonstrated that attentional capture by nontarget singletons is mediated by current task sets. In these studies, spatially nonpredictive singleton cues captured attention (as reflected by spatial cueing effects, i.e., faster responses to subsequent visual search targets presented at cued

Martin Eimer, Monika Kiss, Clare Press, and Disa Sauter, Birkbeck College, University of London.

This research was supported by a grant from the Biotechnology and Biological Sciences Research Council (BBSRC), UK. Martin Eimer holds a Royal Society-Wolfson Research Merit Award. The authors thank Hermann Müller and Ulrich Ansorge for valuable comments.

Correspondence concerning this article should be addressed to Martin Eimer, School of Psychology, Birkbeck College, University of London, Malet Street, London WC1E 7HX, UK. E-mail: m.eimer@bbk.ac.uk locations) only when their features matched the current task set. For example, spatial cueing effects were found for color singleton cues when targets were also color singletons, but not when targets were defined by abrupt onset. Such findings led to the contingent involuntary orienting hypothesis (Folk et al., 1992), which postulates that salient visual objects will capture attention only if they match task-relevant attributes as defined in the current task set.

The hypothesis that attention is captured in a bottom-up fashion by salient visual singletons regardless of their task relevance (e.g., Theeuwes, 1991) appears inconsistent with the finding that spatial cueing effects are eliminated when singleton cues do not share attributes with targets (Folk et al., 1992). To resolve this inconsistency, Theeuwes, Atchley, and Kramer (2000) have argued that although salient singleton stimuli will always capture attention independently of current task sets, attention is rapidly disengaged from stimuli that have no task-set relevant attributes. When this disengagement is completed before the presentation of subsequent target displays, no spatial cueing effects will be observed, as was the case in the studies by Folk and colleagues.

This rapid attentional disengagement hypothesis is based on specific assumptions about the time course of attentional capture, and is therefore difficult to test with behavioral measures alone. Scalp-recorded event-related brain potentials (ERPs) offer a more precise measure of the temporal dynamics of perceptual and attentional processes, and are thus ideally suited to investigate such assumptions. We have recently tested the rapid attentional disengagement hypothesis in an ERP study (Eimer \& Kiss, 2008), where the N2pc component was measured in response to color singleton cues that preceded visual search targets, while task set was manipulated. The N2pc is an enhanced negativity over posterior scalp electrodes contralateral to the side of an attended stimulus, is typically elicited between 180 and $300 \mathrm{~ms}$ after the onset of a visual search array, and is assumed to reflect the attentional selection of candidate target items among distractors in a visual search task (Luck \& Hillyard, 1994a, 1994b; see also 
Eimer, 1996; Girelli \& Luck, 1997; Woodman \& Luck, 1999). Thus, the N2pc can be used as an electrophysiological marker of the presence and time course of attentional capture by salient visual events (see also Brisson \& Jolicœur, 2007; Hickey, McDonald, \& Theeuwes, 2006; Jolicœur, Sessa, Dell'Acqua, \& Robitaille, 2006; Kiss et al., 2007; Kiss, Jolicœur, Dell'Acqua, \& Eimer, 2008a; Kiss, Van Velzen, \& Eimer, 2008b; Mazza, Turatto, Umiltà, \& Eimer, 2007). In our previous study (Eimer \& Kiss, 2008), behavioral spatial cueing effects were accompanied by an early $\mathrm{N} 2 \mathrm{pc}$ in response to red color singleton cues, reflecting rapid attentional capture, when observers had to report the orientation of a uniquely colored red target bar among distractor bars (color task). In contrast, when the task was to report the orientation of a grey target bar presented without distractors, or to report the orientation of a smaller target bar presented among larger distractors, no behavioral cueing effects were found, and no N2pc was triggered to color singleton cues, despite the fact that these were physically identical to the cues shown in the color task. If color singleton cues had initially captured attention regardless of their task relevance, as postulated by Theeuwes et al. (2000), an early N2pc should have been triggered by these cues irrespective of task instructions. The presence of the N2pc in the color task, and its absence when color singleton cues did not match current target features is not in line with the predictions of the rapid attentional disengagement hypothesis proposed by Theeuwes et al. (2000), and thus provides further evidence that attentional capture is strongly mediated by currently active task sets.

In this previous ERP study (Eimer \& Kiss, 2008), we used the N2pc to demonstrate task-set contingent attentional capture by contrasting task conditions where color singleton cues matched both the target dimension (color) and feature (red) with conditions where a different target dimension (onset or size) was task relevant. It has also been demonstrated that attentional capture also depends on top-down task set when task-relevant and irrelevant singleton stimuli differ only with respect to their specific feature value within a given target dimension, such as color. For example, Folk and Remington (1998) found attentional capture effects only for singleton cues that exactly matched the task-relevant feature (e.g., red), but not for nontarget-color singletons (NTCS), suggesting that contingent capture operates at the level of specific stimulus features (but see Folk et al., 1992, Exp. 4, for earlier results that indicated feature-unspecific attentional capture). More recently, Lamy, Leber, and Egeth (2004) observed spatial cueing effects indicative of contingent attentional capture for target-color singleton (TCS) cues, as well as small but reliable inverted cueing effects (i.e., delayed RTs for targets at cued locations) when NTCS cues preceded search displays that contained a color target. These authors suggested that color singletons whose features do not match the currently active task set might trigger a transient location-specific inhibition. In a recent ERP experiment, Lien, Ruthruff, Goodin, and Remington (2008) used the N2pc to further demonstrate that task-set contingent attentional capture by uninformative spatial cues can be mediated at the feature level. Target arrays that included a target-color letter together with one nontarget-color letter and two white letters were preceded by cue arrays containing a target-color cue and a nontarget color item on opposite sides together with two white items. A robust N2pc was observed contralateral to the target-color cue, indicative of featurespecific contingent attentional capture.
While the experiments by Folk and Remington (1998), Lamy et al. (2004), and Lien et al. (2008) have shown that attentional capture by feature singletons is mediated by top-down task set, these results do not necessarily imply that bottom-up factors are entirely irrelevant. The aim of the present study was to investigate two important aspects in which bottom-up salience might still be involved in task-set contingent attentional capture. On the one hand, contingent capture effects are usually found in response to highly salient singleton stimuli (such as the color singleton cues used by Folk and Remington, 1998), thus leaving open the possibility that such effects may be much smaller or even entirely absent for less salient nonsingleton stimuli. If this was the case, bottom-up salience would still be an important factor in task-set contingent attentional capture. To address this issue, Lamy et al. (2004) compared behavioral spatial cueing effects elicited by color singleton cues and by nonsingleton cues (e.g., target-color cues presented in heterogeneous cue displays together with items in five other colors) that both matched the target-defining color (e.g., red), and found little evidence for systematic differences in the magnitude of attentional capture. Furthermore, Lien et al. (2008) observed an N2pc to target-color cues, indicative of attentional capture, despite the fact that these cues were accompanied by equally salient nontarget-color cues on the opposite side. These results suggest that high bottom-up salience is not a necessary condition for task-set contingent capture. However, capture might still be elicited more effectively, or faster, by salient stimuli such as color singletons than by less salient nonsingleton stimuli. One aim of the present study was to directly compare behavioral and electrophysiological markers of contingent capture triggered in response to these two types of stimuli.

Another possible role for bottom-up salience in contingent attentional capture was suggested by the results of Lamy et al. (2004). These authors observed inverted spatial cueing effects (i.e., delayed responses to targets at cued locations) for NTCS cues (e.g., red cues when participants searched for green targets; see also Lamy \& Egeth, 2003, for similar effects with irrelevantdimension cues), and postulated that perceptually salient stimuli that do not match the current top-down task set are actively inhibited. If such stimuli do indeed trigger location-specific inhibition, this would again imply that bottom-up salience plays an important, albeit indirect, role in contingent attentional capture. However, the hypothesis that salient nontarget feature singletons are subject to top-down inhibition is so far supported only by small, and not always reliable inverted behavioral spatial cueing effects in response to these stimuli. The second aim of the present study was to obtain new and more direct electrophysiological evidence for the location-specific inhibition of salient NTCS.

The general procedures used were similar to those employed by Lamy et al. (2004). In Experiment 1, participants searched for a known color target in circular visual search arrays that contained six horizontal or vertical bars in six different colors (see Figure 1). Half of the participants had to report the orientation of the red bar, whereas blue bars served as targets for the other half of participants. Because these target bars were not unique color singletons, they could not be detected by using a singleton search mode (i.e., a search for any feature discontinuity irrespective of its value, see Bacon \& Egeth, 1994). Instead, participants had to adopt a more specific feature search mode to find the bar in the task-relevant color. One of three cue arrays was presented $250 \mathrm{~ms}$ before the 

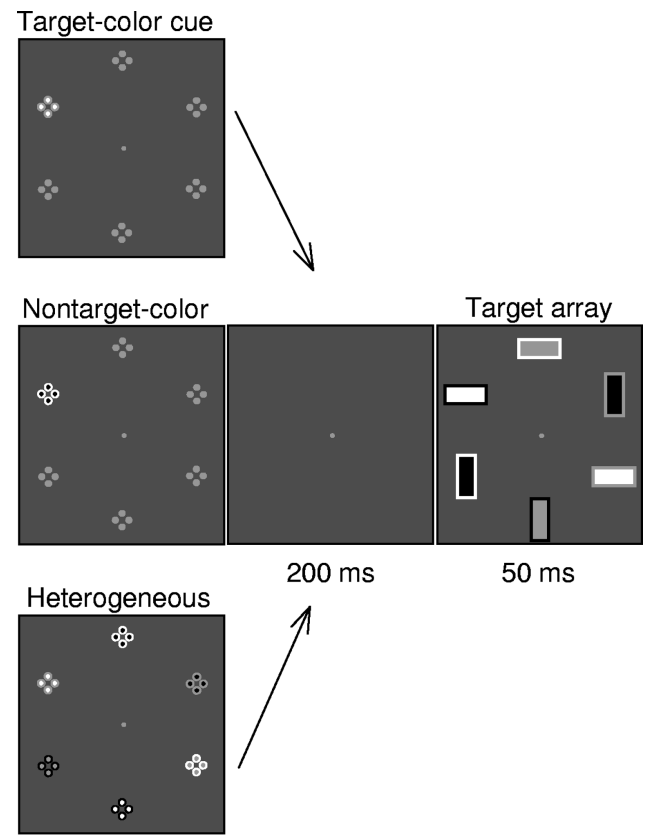

$50 \mathrm{~ms}$

Figure 1. Examples of trial sequences for the three cue conditions in Experiment 1. In all trials, a cue array was presented for $50 \mathrm{~ms}$ and was followed after a blank interval of $200 \mathrm{~ms}$ by a target array $(50 \mathrm{~ms}$ in duration). Different groups of participants had to report the orientation of either the red or the blue bar stimulus in the target array, and this Figure shows trials for participants searching for a red target. Top panel: TCS cue array. Middle panel: NTCS cue array. Bottom panel: HTC cue array. Each bar in the target array had a different color, as indicated by different combinations of bar shading and outline shading (white, grey, or black). Red is depicted as white with grey outline and blue is depicted as black with white outline. Grey distractor elements in the cue array are depicted without an outline. In Experiment 2, the duration of the blank interval separating cue and target arrays was reduced to $150 \mathrm{~ms}$, and no HTC cue arrays were included.

onset of each target search display (Figure 1). The TCS cue array contained one small circular set of red dots (for participants searching for red targets) or blue dots (for participants searching for blue targets) together with five sets of grey dots. The NTCS cue array was physically identical, except that the color of the singleton did not match the target color (i.e., it was blue for participants searching for red targets, or vice versa). The heterogeneous targetcolor (HTC) cue array contained one set of dots in the target color (red or blue) together with five sets of dots in five different nontarget colors. To identify electrophysiological markers of attentional capture (as well as top-down inhibition of capture, see below), ERPs were measured relative to the onset of these cue arrays.

To confirm that task-set contingent capture operates at the level of specific stimulus features within the color dimension, the TCS cue condition was compared to the NTCS cue condition. These two conditions are physically equivalent (i.e., both contain a red or blue singleton cue among grey items), and only differ with respect to the task relevance of the singleton cue color. For the TCS cue condition, behavioral spatial cueing effects indicative of atten- tional capture (i.e., faster RTs to targets at cued as compared to uncued locations) should be observed, and capture should also be reflected by the presence of an early $\mathrm{N} 2 \mathrm{pc}$ component in response to the cue array, analogous to our previous findings (Eimer \& Kiss, 2008). If contingent attentional capture was mediated at the level of specific target-defining stimulus features, as suggested by the behavioral results of Folk and Remington (1998) and Lamy et al. (2004), as well as by the N2pc results of Lien et al. (2008), no capture should be triggered by NTCS cues, and no N2pc should be triggered in response to these cues. In contrast, if attention was initially captured by NTCS but was then rapidly disengaged, as postulated by Theeuwes et al. (2000), an early but transient N2pc should be observed in response to these stimuli.

To assess the possible role of bottom-up salience in facilitating task-set contingent attentional capture, we compared behavioral performance and N2pc components to TCS cues and HTC cue arrays. Although both types of cue arrays include one item in the task-relevant color, they differ in terms of the bottom-up salience of this item. If capture is exclusively mediated by top-down task set, with no role at all for bottom-up salience, behavioral spatial cueing effects should be equivalent for both types of cue arrays, and N2pc components of similar amplitudes and latencies should be elicited. In contrast, if bottom-up salience modulates task-set contingent attentional capture (e.g., by facilitating the selection of task-set matching singleton stimuli relative to matching nonsingletons), behavioral cueing effects should be larger for singleton cues, and the N2pc component should be delayed and attenuated for heterogeneous as compared to singleton cues.

Another analysis was conducted to investigate whether cue arrays that contain a NTCS cue trigger top-down inhibition, as suggested by Lamy et al. (2004). Top-down processes involved in the strategic monitoring and inhibitory control of perceptual, cognitive, and response-related processes, such as observed with Eriksen flanker interference tasks (e.g., Coles, Gratton, Bashore, Eriksen, \& Donchin, 1985), Stroop tasks (e.g., Liotti, Woldorff, Perez, \& Mayberg, 2000), or Go/Nogo tasks (e.g., Eimer, 1993) are commonly associated with anterior negativities in the time range of the N2 component (see Folstein \& Van Petten, 2008, for a recent review). To identify an ERP marker of top-down inhibition of attentional capture, we compared nonlateralized ERPs obtained at anterior electrodes in response to physically equivalent targetcolor and NTCS cues. ${ }^{1}$ While the former match the target color and should thus not be subject to inhibition, the latter might be inhibited because of their mismatch with the task-relevant color. If top-down inhibition did indeed occur, this should be revealed by ERPs as an enhanced anterior N2 component in response to NTCS cues. Experiment 2 (described in more detail below) was conducted to address the hypothesis that nontarget-color singleton cues are subject to location-specific inhibition by measuring not only the anterior $\mathrm{N} 2$ component in response to these cues, but also the N2pc to subsequent target arrays.

\footnotetext{
${ }^{1}$ This comparison was restricted to TCS and NTCS cues, and did not include HTC cue arrays, because the latter differ in terms of their low-level physical features from the two former types of cues. Such low-level perceptual differences usually have a substantial impact on nonlateralized ERP components that would be difficult to disentangle from any ERP differences associated with top-down inhibition.
} 


\section{Experiment 1}

\section{Method}

Participants. Fifteen volunteers took part in the experiment. Two participants were excluded because of excessive alpha activity at posterior electrodes. One further participant was excluded because of an inability to perform the visual search task as instructed. All of the remaining 12 participants (mean age 28.2 years, 5 male) had normal or corrected vision, and all but 1 were right-handed.

Stimuli and procedure. Cue and target arrays consisted of six elements placed at equidistant positions along the circumference of an imaginary circle, at a distance of $4.3^{\circ}$ from a central fixation point (Figure 1). Target search arrays contained six colored horizontal or vertical bars (each subtending $1.3^{\circ} \times 0.5^{\circ}$ ). The orientation of each bar (horizontal or vertical) varied randomly across trials. Each bar had a different color (CIE chromaticity coordinates $\mathrm{x} / \mathrm{y}$ for red .574/.337; blue .149/.068; green .285/.554; yellow .394/.513; turquoise .213/.307; purple .281/.139). Participants had to report the orientation of the bar in the target color by pressing one of two vertically arranged response keys with their left or right index fingers. After half of the blocks, assignment of fingers to response keys was reversed. For 6 participants, the red bar served as target, while the blue bar was the target for the other 6 participants. This target-color bar was presented in random order and with equal probability at one of the four lateral locations, but never at the top or bottom position. ${ }^{2}$ The remaining five positions were randomly filled with horizontal or vertical bars in each of the five nontarget colors.

Target search arrays were preceded by circular cue arrays that contained six sets of four closely aligned dots, with each set subtending $0.8^{\circ} \times 0.8^{\circ}$ visual angle (see Figure 1 ). Three different types of cue arrays were presented. In the TCS cue condition, one set of dots matched the current target color (red or blue for different participants), whereas all other sets of dots were grey (CIE coordinates $x / y$.280/.312). In the NTCS cue condition, one set of dots was blue when the target was red, or red when the target was blue, whereas all other sets of dots were grey. In the HTC cue condition, one set of dots matched the current target (red or blue for different participants), whereas each of the other sets of dots was randomly assigned one of the distractor colors green, yellow, turquoise, purple, and blue (if the target was red) or red (if the target was blue). As was the case for target bars (see above), color singleton cues and target-color cues in heterogeneous cue arrays were presented randomly and equiprobably at one of the four locations left and right of the fixation point, but never at the top or bottom position.

Participants completed 12 experimental blocks with 96 trials each, resulting in a total of 1,152 trials. All three cue conditions were randomly intermixed within each block. Cue arrays were presented for $50 \mathrm{~ms}$, followed by a blank 200-ms interstimulus interval and a search array presented for $50 \mathrm{~ms}$. Intertrial interval was $1,500 \mathrm{~ms}$. Color singleton cues and target-color cues in heterogeneous cue arrays were uninformative with respect to target location. All stimuli were approximately isoluminant $\left(\sim 11 \mathrm{~cd} / \mathrm{m}^{2}\right)$, and were presented on a 17 -inch computer monitor against a black background.
Electrophysiological recording and data analysis. Electroencephalogram (EEG) was recorded with a Brainamps DC amplifier with a bandpass of 0 to $40 \mathrm{~Hz}$ and a sampling rate of $500 \mathrm{~Hz}$ from $23 \mathrm{Ag}-\mathrm{AgCl}$ electrodes mounted in an elastic cap according to the extended 10 to 20 system at scalp sites Fpz, F7, F3, Fz, F4, F8, FC5, FC6, T7, C3, Cz, C4, T8, CP5, CP6, P7, P3, Pz, P4, P8, PO7, $\mathrm{PO} 8$, and Oz. Horizontal eye movements (HEOG) were measured from two electrodes placed at the outer canthus of each eye. The right earlobe was recorded as an additional channel. All electrodes were referenced to the left earlobe and re-referenced offline to averaged earlobes. Impedances were kept below $8 \mathrm{k} \Omega$. All off-line analyses of EEG data were conducted with Brainvision Analyser software. The EEG was epoched into 400-ms segments from 100 $\mathrm{ms}$ before cue onset to $300 \mathrm{~ms}$ after cue onset. Trials with saccades (voltage exceeding $\pm 25 \mu \mathrm{V}$ in the HEOG channel), eye blinks (exceeding $\pm 60 \mu \mathrm{V}$ at Fpz), or muscular artifacts (exceeding \pm $80 \mu \mathrm{V}$ at any other electrode) were excluded from analysis. Trials with incorrect responses, and with RTs to targets that exceeded the mean RT for this specific trial condition by more than $3.5 S D$ s were also excluded from all analyses ( $0.6 \%$ of all trials).

ERPs in response to the cue arrays were averaged relative to a 100-ms precue baseline for each combination of cue condition (TCS cue, NTCS, and HTC cue) and cue position (left vs. right hemifield), collapsed across all four possible target locations.

The N2pc component was quantified by measuring mean ERP amplitudes at lateral posterior electrodes $\mathrm{PO} / \mathrm{PO} 8$ contralateral and ipsilateral to the side of the color cue in a time window 180 to $280 \mathrm{~ms}$ after cue onset. Mean amplitudes were analyzed in threeway analyses of variance (ANOVAs) for the factors cue condition, contralaterality (hemisphere contralateral vs. ipsilateral to the color cue), and cue position. The frontal N2 component was measured within the 170- to 270-ms time interval after cue onset at midline electrode sites $\mathrm{Fpz}, \mathrm{Fz}$, and $\mathrm{Cz}$, and at lateral frontal electrodes F3/F4 and F7/F8, for the target-color and NTCS cue arrays. ANOVAs with cue condition as a two-level factor (TCS vs. NTCS) were conducted separately for midline electrodes, with factor electrode $(\mathrm{Fpz}, \mathrm{Fz}$, and $\mathrm{Cz}$ ), and for lateral electrodes, with factors hemisphere (left vs. right) and electrode site (F3/4 vs. F7/8).

RTs and error rates were analyzed in a two-way ANOVA with the factors cue condition and cue-target position (same vs. different). In all analyses, Greenhouse-Geisser corrections for nonsphericity were applied where appropriate. Preliminary behavioral and ERP analyses were conducted with the between-subject factor target color (red vs. blue). Because these analyses revealed no systematic differences between these two groups, this factor was not included in any of the subsequent analyses.

\section{Results}

Behavior. Correct RTs (line graphs) and error rates (bar graphs) observed in Experiment 1 for all three cue conditions are presented in Figure 2 (left panel), separately for trials in which color cues and targets appeared at the same location or at different

\footnotetext{
${ }^{2}$ Color cues and color targets were always presented at one of the four lateral positions because the N2pc component is a lateralized ERP response that can only be measured in response to stimuli in the left or right visual field, but not for stimuli located on the vertical meridian.
} 


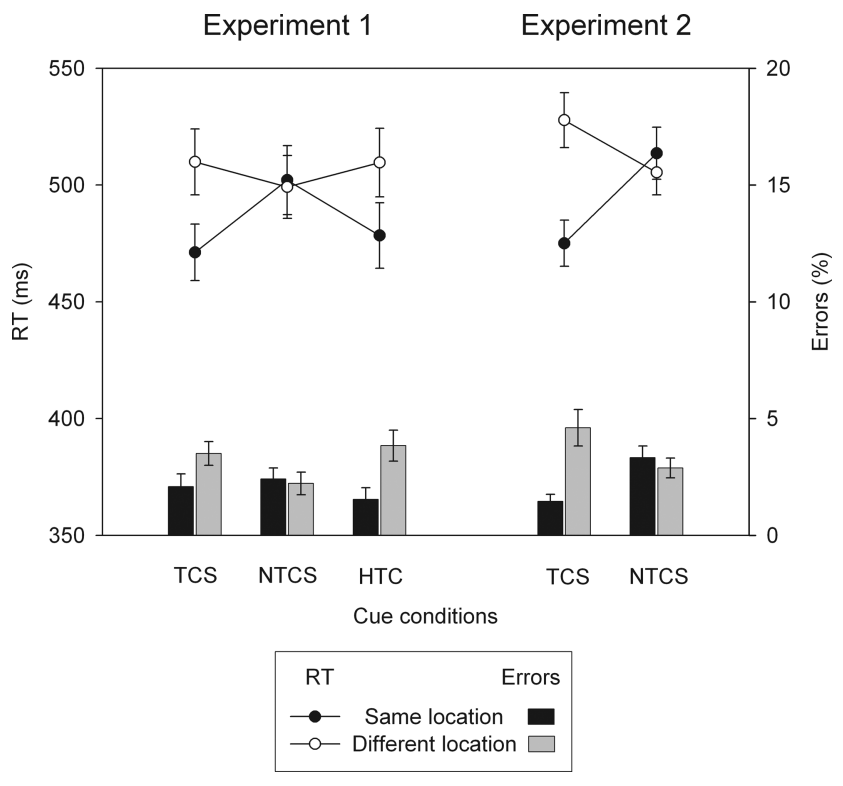

Figure 2. Response times (RT; lines) and error rates (bars) in Experiments 1 and 2 for each condition (target-color singleton [TCS], nontargetcolor singleton [NTCS], and heterogeneous target color [HTC] cue) on trials where target-color cues or nontarget-color singleton cues and targets were presented at the same location or at different locations.

locations. Spatial cueing effects indicative of attentional capture were elicited in response to both TCS and HTC cues, and these effects were similar in magnitude. With TCS cues, RTs were 39 $\mathrm{ms}$ faster for targets at cued versus uncued locations, and this difference was $31 \mathrm{~ms}$ with HTC cues. In contrast, a small RT advantage for uncued locations ( $3 \mathrm{~ms}$ ) was obtained in response to NTCS cues. This was confirmed by the omnibus ANOVA, where main effects of cue condition, $F(2,22)=5.2, p<.05, \eta_{\mathrm{p}}^{2}=.319$, and cue-target position, $F(1,11)=28.0, p<.001, \eta_{\mathrm{p}}^{2}=.718$, were accompanied by an interaction of cue condition and cuetarget position, $F(2,22)=16.8, p<.001, \eta_{\mathrm{p}}^{2}=.604$.

RTs for the TCS cue and HTC cue conditions were directly compared in a separate ANOVA with cue condition as a two-level factor. There was a main effect of cue-target position, $F(1,11)=$ $26.4, p<.001, \eta_{\mathrm{p}}^{2}=.706$, but no main effect of cue condition. The Cue condition $\times$ Cue-target position interaction was marginally significant, $F(1,11)=4.5, p=.058, \eta_{\mathrm{p}}^{2}=.29$, reflecting the slightly larger cueing effects observed for singleton relative to HTC cues. Two further ANOVAs were conducted to compare spatial cueing effects in the NTCS cue condition with effects in the two other cue conditions. In both analyses, significant interactions of cue condition with cue-target position were obtained, both $F(1$, 11) $>16.8$, both $p<.01$, both $\eta_{\mathrm{p}}^{2}>.605$, demonstrating that spatial cueing effects in response to NTCS cues differed from cueing effects obtained with target-color cues. Follow-up $t$ tests confirmed spatial cueing effects for HTC and TCS cues, both $t(11)=5.0$, both $p<.001$. However, the small RT advantage for targets at uncued locations observed for NTCS cues was not significant $(t<1)$.

Error rates were generally low and showed a similar pattern as was found for RTs, with fewer errors in response to targets at cued locations in the TCS and HTC cue conditions (see Figure 2). The omnibus ANOVA found a main effect of cue-target position, $F(1$, $11)=7.3, p<.05, \eta_{\mathrm{p}}^{2}=.399$, and an interaction of cue condition with cue-target position, $F(2,22)=4.8, p<.05, \eta_{\mathrm{p}}^{2}=.306$. When TCS and HTC cue conditions were compared in a separate ANOVA, a main effect of cue-target position was obtained, $F(1$, $11)=12.3, p<.01, \eta_{\mathrm{p}}^{2}=.529$, in the absence of any interaction between cue condition and cue-target position $(F<1)$, indicating that cueing effects on error rates were similar for both cue conditions. As for RTs, two analogous ANOVAs were conducted to compare spatial cueing effects on error rates in the NTCS cue condition with effects in the two other cue conditions. In both analyses, significant interactions of cue condition with cue-target position were obtained, both $F(1,11)>7.3$, both $p<.02$, both $\eta_{\mathrm{p}}^{2}>.4$, demonstrating systematic differences in the impact of spatial cueing on error rates for target-color relative to nontarget-color cues. Follow-up $t$-tests confirmed fewer errors on same-location as compared to different-location trials for both singleton and HTC cue conditions, $t(11)=2.6$ and 3.2 , both $p<.05$, but found no significant difference for NTCS cues $(t<1)$.

\section{ERP Data}

N2pc component. Figure 3 shows ERP waveforms measured in Experiment 1 in response to the color cue arrays at posterior electrodes PO7/8 contralateral and ipsilateral to the visual field where the color cue was presented, separately for each cue condition. To highlight the time course, magnitude, and topography of the N2pc in response to the three different cue array types, Figure 3 also shows difference waveforms computed by subtracting ipsilateral from contralateral ERP activity, and scalp distribution maps of the N2pc for TCS cues and for HTC cues. An N2pc component was clearly triggered in response to both TCS cues and HTC cue arrays. Although the onset of the N2pc appears similar for both types of cues, N2pc peak amplitude was larger for the TCS as compared to the HTC cue. In marked contrast, no N2pc appears to be present in response to NTCS cues.

In the omnibus ANOVA of ERP mean amplitudes obtained in the 180 - to $280-\mathrm{ms}$ postcue time interval, the presence of an N2pc component was confirmed by a main effect of contralaterality, $F(1$, $11)=32.5, p<.001, \eta_{\mathrm{p}}^{2}=.747$. The fact that the $\mathrm{N} 2 \mathrm{pc}$ differed across cue array types was substantiated by an interaction between cue condition and contralaterality, $F(2,22)=23.1, p<.001$, $\eta_{\mathrm{p}}^{2}=.677$. In separate ANOVAs conducted for each cue condition, a significant effect of contralaterality was observed for both TCS cues, $F(1,11)=28.8, p<.001, \eta_{\mathrm{p}}^{2}=.723$, and HTC cues, $F(1$, $11)=33.4, p<.001, \eta_{\mathrm{p}}^{2}=.752$, confirming the presence of an $\mathrm{N} 2 \mathrm{pc}$ in these two conditions. In contrast, there was no indication that an N2pc was present in the nontarget-color cue condition (effect of contralaterality: $F<1$ ).

To investigate N2pc amplitude differences between TCS and HTC cue conditions, an additional ANOVA was conducted on the data from these two conditions only. A main effect of contralaterality, $F(1,11)=32.6, p<.001, \eta_{\mathrm{p}}^{2}=.748$, was accompanied by an interaction of cue condition and contralaterality, $F(1,11)=6.9$, $p<.05, \eta_{\mathrm{p}}^{2}=.384$, confirming that the N2pc elicited by TCS cues 
TCS
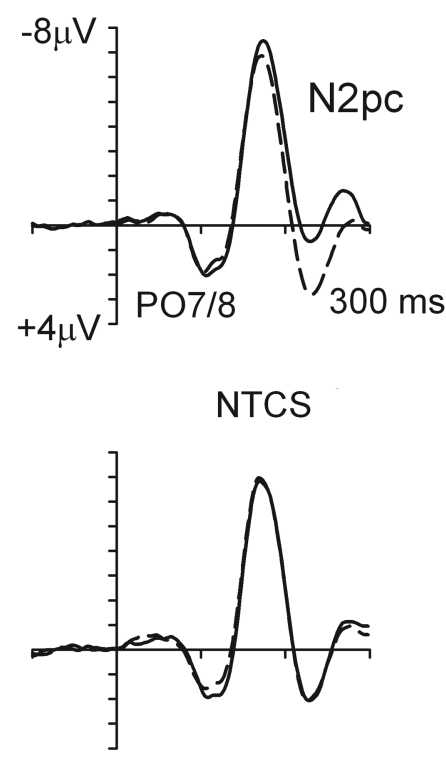

HTC

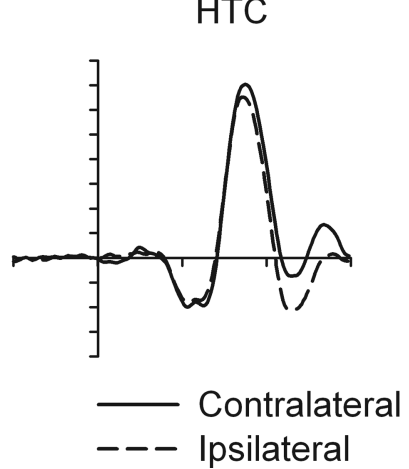

NTCS

Difference waves

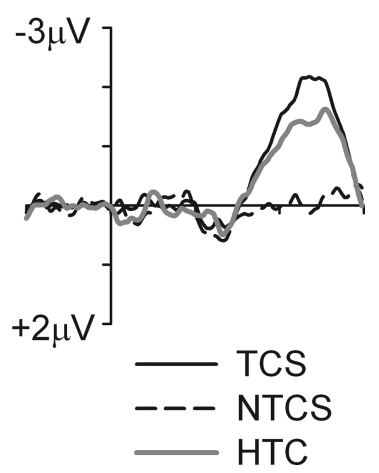

Figure 3. Left panels: Event-related brain potentials (ERPs) elicited in Experiment 1 in the 300-ms interval after cue onset in the target-color singleton (TCS) cue condition, nontarget-color singleton (NTCS) cue condition, and heterogeneous target color (HTC) cue condition at posterior electrode sites PO7/8 contralateral (solid lines) and ipsilateral (dashed lines) to the visual hemifield where the color cue was presented. Each tick on the $\mathrm{x}$ - and $\mathrm{y}$-axes represents steps of $100 \mathrm{~ms}$ and $1 \mu \mathrm{V}$, respectively. Top right panel: Difference waveforms obtained by subtracting ipsilateral from contralateral ERPs in the TCS cue condition (solid black line), NTCS cue condition (dashed line), and HTC cue condition (solid grey line). Bottom right panels: Topographical maps (back views) of N2pc scalp distribution (180-to 280-ms postcue) for TCS and HTC cue arrays. Maps were constructed by spherical spline interpolation (see Perrin, Pernier, Bertrand, \& Echallier, 1989) after mirroring the ipsilateralcontralateral difference waveforms to obtain symmetrical voltage values for both hemispheres. The N2pc appears as negative voltage (-) over the left hemisphere and as positive voltage $(+)$ over the right hemisphere.

was larger than the N2pc in response to the HTC cue array (see Figure 3).

Frontal N2 component in response to color singleton cue arrays. Figure 4 (left) shows nonlateralized ERP waveforms obtained in Experiment 1 at anterior electrode sites in response to cue arrays that contained a color singleton, as a function of whether this singleton matched the current target color (TCS cue, solid lines) or not (NTCS cue, dashed lines). The scalp distribution of the ERP amplitude difference between NTCS and TCS cues obtained in the 170- to 270-ms postcue interval is also shown in Figure 4. As can be seen in Figure 4, a marked frontal N2 component was triggered in response to NTCS cues, in line with the hypothesis that these stimuli are subject to top-down inhibition. Analyses of ERP mean amplitudes obtained at midline electrodes $\mathrm{Fpz}, \mathrm{Fz}$, and $\mathrm{Cz}$ in the 170 - to $270-\mathrm{ms}$ interval after cue onset revealed a main effect of cue condition (TCS vs. NTCS cue), $F(1$, $11)=8.2, p<.05, \eta_{\mathrm{p}}^{2}=.426$, confirming the presence of an enhanced N2 for NTCS cues. An analogous effect of cue condition, $F(1,11)=19.1, p<.001, \eta_{\mathrm{p}}^{2}=.635$, was obtained in the analysis for lateral frontal electrode pairs F3/4 and F7/8. Although the topographical map shown in Figure 4 (bottom midleft) suggests that this $\mathrm{N} 2$ effect might be slightly more pronounced over the right hemisphere, this analysis revealed no significant interaction between cue condition and hemisphere, $F(1,11)=2.7, p=.128$.

\section{Discussion}

The results from Experiment 1 provide further evidence for the hypothesis that task-set contingent attentional capture is featurespecific: Behavioral spatial cueing effects were accompanied by an N2pc component only when cue arrays contained a TCS cue but not when a NTCS cue was presented, despite the fact that both types of arrays were equivalent in terms of their bottom-up salience. These observations are in line with results from previous behavioral (Folk and Remington, 1998; Lamy et al., 2004) and ERP studies (Lien et al., 2008). In addition, and importantly, Experiment 1 found no evidence for an initial capture and rapid disengagement of attention in response to NTCS cues, as postulated by Theeuwes et al. (2000). If this had been the case, the onset and early phase of the N2pc component should have been similar for TCS and NTCS cues, with differences because of task relevance only emerging later. However, this pattern of results was not observed.

One main objective of Experiment 1 was to directly contrast the roles of top-down task set and bottom-up salience in attentional capture by comparing the $\mathrm{N} 2 \mathrm{pc}$ in response to cues that match the current target color when their bottom-up salience is high (TCS cues) or low (HTC cues). While Lien et al. (2008) have recently employed $\mathrm{N} 2 \mathrm{pc}$ measures to demonstrate contingent attentional capture by nonsingleton color cues, no previous study has directly compared N2pc components to high- versus low-salience cues. Behavioral spatial cueing effects were obtained in Experiment 1 for both types of cues. Although these effects were slightly larger for singleton cues, this difference was only a nonsignificant trend, thus suggesting that singleton and nonsingleton target-color cues were similarly effective in capturing attention (see also Lamy et al., 2004, for comparable results). This conclusion was supported by the ERP results. Despite their salience difference, TCS cues and HTC cues triggered remarkably similar N2pc components (see Figure 3), demonstrating that bottom-up salience does not play a major role in determining task-set contingent attentional capture. However, there was a small but reliable amplitude difference, 


\section{Experiment 1}
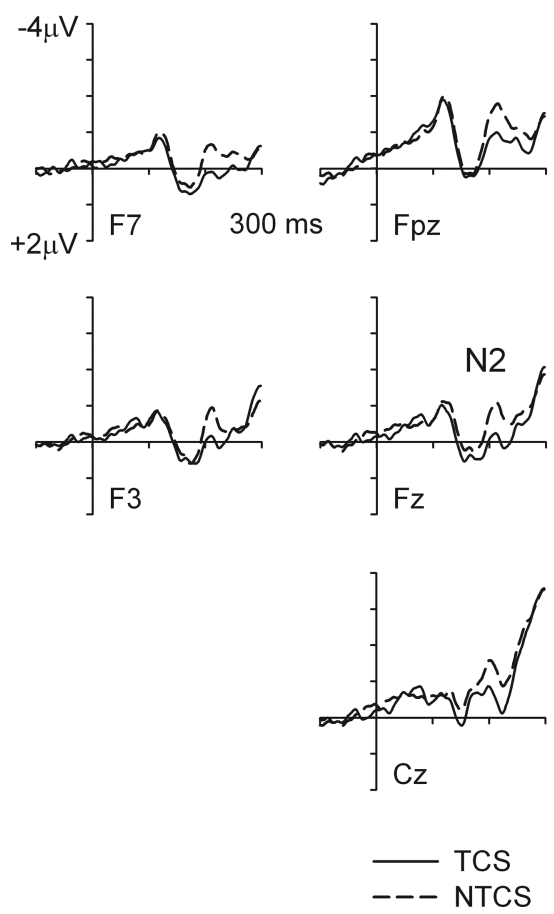
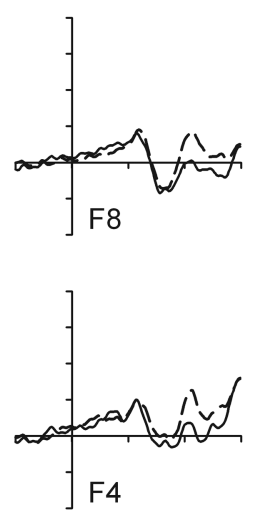

Difference map

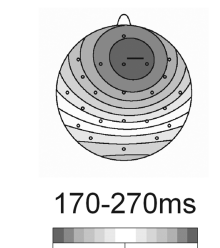

$-0.6 \mu \mathrm{V}+0.6 \mu \mathrm{V}$
Experiment 2
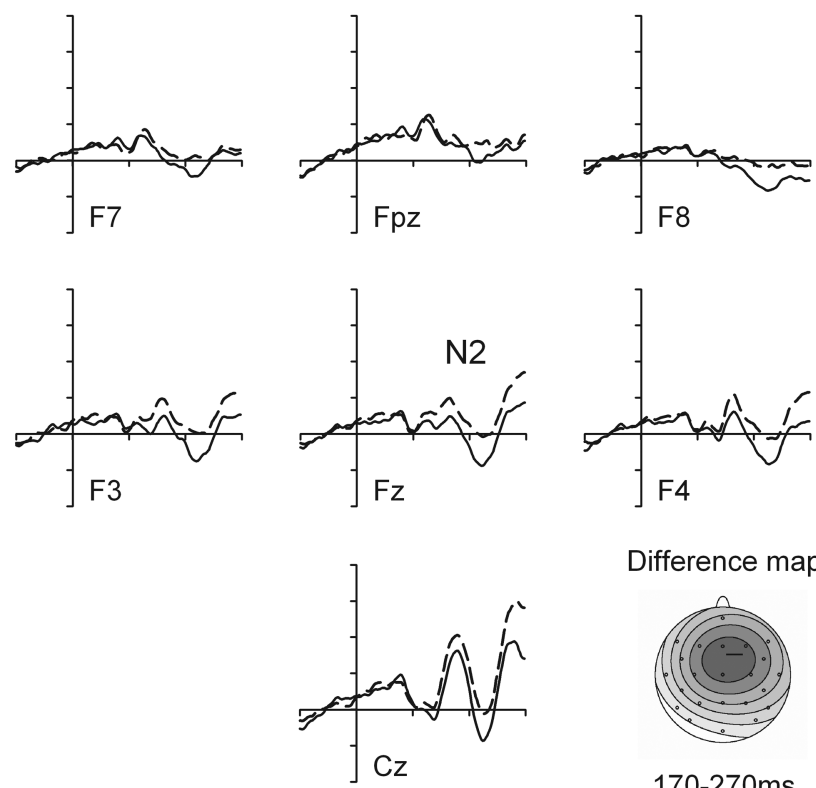

Difference map

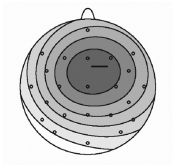

170-270ms

$-0.6 \mu \mathrm{V} \quad+0.6 \mu \mathrm{V}$

Figure 4. Event-related brain potentials (ERPs) elicited in Experiment 1 (left) and Experiment 2 (right) at frontocentral electrode sites in the target-color singleton (TCS) (solid lines) and nontarget-color singleton (NTCS) (dashed lines) cue conditions. Topographical maps (top views) show the distribution of the N2 enhancement for NTCS cues in both experiments. Maps were constructed by spherical spline interpolation of difference waveforms obtained by subtracting ERPs to TCS cues from ERPs to NTCS cues.

with larger N2pc amplitudes in response to TCS cues. Such amplitude differences often indicate that the underlying cognitive process (here: attentional capture) is triggered more consistently across trials in one condition than in the other. If TCS cues had captured attention on virtually all trials, whereas HTC cues had failed to do so on a small subset of trials, moderate N2pc amplitude differences, but no onset latency differences, between these two cue conditions would result.

Although the N2pc amplitude difference between TCS cues and HTC cues indicates that bottom-up salience may have had a modulating effect on the efficiency of task-set contingent attentional capture, this difference was very small, despite the large contrast in the perceptual salience of these two types of cues. This observation underlines the dominance of top-down task over salience in attentional capture. The differential impact of top-down task set and bottom-up salience on capture can also be evaluated by directly comparing the N2pc in response to HTC cues and NTCS cues (Figure 3). The former are task relevant but not salient, whereas the latter are task irrelevant but score high in terms of their salience. The fact that an N2pc was absent for NTCS cues demonstrates that bottom-up salience in itself is not sufficient to trigger attentional capture. In contrast, the fact that the N2pc was present for HTCS cues shows that task relevance in itself is sufficient for attentional capture to occur, even when bottom-up salience is low.

However, the observation that NTCS cues failed to trigger attentional capture, as reflected by the N2pc component, does not neces- sarily imply that the bottom-up salience of these stimuli had no impact at all on their processing. The absence of attentional capture for these stimuli might have been the result of active top-down inhibition, as suggested previously by Lamy et al. (2004). The second main aim of Experiment 1 was to identify a possible ERP marker for this type of inhibition of irrelevant singletons by comparing ERPs elicited at anterior electrodes in response to NTCS and TCS cues. These two types of cue arrays were identical in terms of their bottom-up salience (i.e., both contained a red or blue color singleton stimulus among grey distractor items), but may differ with respect to the presence or absence of top-down inhibition. An enhanced N2 component was found in response to NTCS cues, and the polarity, latency, and anterior scalp distribution of this effect (as shown in Figure 4) was remarkably similar to modulations of the $\mathrm{N} 2$ that have been observed previously in a variety of tasks that involve top-down control, inhibition, and conflict resolution with respect to perceptual, cognitive and response-related processes (see Folstein \& Van Petten, 2008, for a detailed review of links between the anterior N2 component and top-down control). Such similarities suggest that the anterior N2 modulation found in the present experiment might represent an electrophysiological marker of the top-down inhibition of color singletons whose features do not match the current task set.

Although such an interpretation appears plausible, additional and more direct evidence for the presence of such an inhibitory mechanism would be desirable. As the presence of an anterior N2 component to NTCS cues is a new finding, the reliability of this effect needs to be confirmed. It is also important to note that the 
existence of such an inhibitory mechanism was originally postulated by Lamy et al. (2004) on the basis of inverted behavioral spatial cueing effects (i.e., faster RTs for targets at uncued locations) observed for NTCS cues. However, no reliable inverted cueing effects were observed for NTCS cues in Experiment 1. This may have been because of the fact that the stimulus onset asynchrony (SOA) between cue and target array was relatively long (250 ms). In the Lamy et al. (2004) study, inverted spatial cueing effects were minimal or entirely absent when the cue-target SOA was $250 \mathrm{~ms}$, suggesting that top-down inhibition is a transient phenomenon that dissipates rapidly when SOA is increased. When designing Experiment 1, a longer SOA was chosen to allow for the possibility that the N2pc was substantially delayed in response to HTC cue arrays (which turned out not to be the case), given the necessity to reliably measure cue-induced N2pc components before the emergence of visual ERP components triggered by the subsequent target array. In Experiment 2, the SOA between cue and target arrays was reduced to obtain more direct behavioral as well as additional electrophysiological evidence for the presence of a location-specific inhibitory mechanism in attentional capture.

\section{Experiment 2}

The general procedure used in Experiment 2 was analogous to Experiment 1. Because the specific aim of Experiment 2 was to investigate the hypothesis that salient but task-irrelevant visual events are subject to top-down inhibition, the HTC cue condition of Experiment 1 was omitted. Importantly, the SOA between cue and target array onset was reduced from 250 to 200 ms. Shortening the interval between cue and target arrays should not affect the presence of positive behavioral cueing effects for TCS cues, but should make it more likely that nontarget-color cues yield inverted cueing effects (see Lamy et al., 2004), indicative of transient location-specific top-down inhibition. In this case, ERP measures can be used to gain further insights into the neural basis of this effect. In Experiment 2, N2pc components were measured in response to both types of singleton cue arrays, as well as in response to subsequent target arrays. If NTCS cues trigger location-specific inhibition, this might affect the attentional selection of targets that immediately follow these cues, and thus be reflected in systematic latency differences in N2pc components triggered by such targets. More specifically, the target N2pc should be delayed on trials where targets are preceded by NTCS cues at the same location, relative to trials where cues and targets are presented in opposite hemifields, and also relative to trials where they appear in the same hemifield, but at different locations. Another aim of Experiment 2 was to confirm the observation of Experiment 1 that an anterior N2 indicative of top-down inhibition is elicited when color singleton cues do not match the current task set.

\section{Method}

Participants. Twelve new participants (5 male, mean age 26.8 years) were recruited. All of them were right-handed and had normal or corrected vision.

Stimuli, procedure, and data analysis. Stimulus material and procedures were identical to Experiment 1, except that the cue- target SOA was shortened to $200 \mathrm{~ms}$, and only the TCS and NTCS cue conditions were included. Participants completed 10 experimental blocks (96 trials per block), and both cue conditions were randomly intermixed within each block.

Data analysis procedures were analogous to Experiment 1. Exclusion of trials with outlier RTs resulted in the removal of $0.7 \%$ of all trials. In addition to the analyses of N2pc and anterior N2 components triggered by cue arrays, another analysis was conducted to examine the onset latency of the target-elicited N2pc in the nontarget-color cue condition as a function of cue-target position. N2pc onset latencies to targets were determined separately for trials where cues and targets where presented at the same location, trials where they were presented at different locations in the same hemifield, and trials where they appeared in opposite hemifields. They were calculated with the jackknife-based procedure described by Ulrich and Miller (2001). This procedure estimates onset latencies based on grand averages computed from subsamples of averaged ERP waveforms obtained by successively excluding one participant from the original sample. The onset latency of the $\mathrm{N} 2 \mathrm{pc}$ component was defined as the time point where the voltage measured in subsamples exceeded $40 \%$ of the peak amplitude. For all N2pc latency comparisons between different types of trials, $F$ values were corrected (indicated with the label $F_{\mathrm{c}}$ ) according to the formula described by Ulrich and Miller (2001).

\section{Results}

Behavior. As in Experiment 1, spatial cueing effects were observed for the TCS cue condition, with faster RTs on trials where cues and targets were presented at the same location (see Figure 2, right panel). In contrast, a small inverted cueing effect was present in the NTCS cue condition. Statistical analyses found main effects of cue condition, $F(1,11)=5.5, p<.05, \eta_{\mathrm{p}}^{2}=.335$, cue-target position, $F(1,11)=45.7, p<.001, \eta_{\mathrm{p}}^{2}=.806$, and an interaction of cue condition and cue-target position, $F(1,11)=$ 53.2, $p<.001, \eta_{\mathrm{p}}^{2}=.829$. Follow-up $t$ tests confirmed the presence of a large positive spatial cueing effect $(53 \mathrm{~ms})$ for the TCS cue, $t(11)=8.0, p<.001$. Importantly, and in contrast to Experiment 1, there was now a reliable reversed cueing effect $(-8$ $\mathrm{ms}$ ) for the NTCS cue condition, $t(11)=-2.3, p<.05$.

A similar pattern was found for error rates. There was a main effect of cue-target position, $F(1,11)=5.8, p<.05, \eta_{\mathrm{p}}^{2}=.343$, and an interaction of cue condition and cue-target position, $F(1$, $11)=15.3, p<.01, \eta_{\mathrm{p}}^{2}=.582$. Follow-up $t$ tests confirmed that in the TCS cue condition, error rates were lower on trials where targets appeared at cued locations than on trials where cues and targets appeared at different locations, $t(11)=3.4$, $p<.01$. No cueing effects on error rates were found for the NTCS cue condition $(t<1)$.

\section{ERP Data}

$N 2 p c$ component in response to cue arrays. Figure 5 shows ERPs measured in Experiment 2 in response to TCS and NTCS cues at posterior electrodes $\mathrm{PO} / / 8$ contralateral and ipsilateral to the visual field of the cues, together with difference waveforms computed by subtracting ipsilateral from contralateral ERPs, and scalp distribution maps of the N2pc for the TCS cue condition. 
TCS

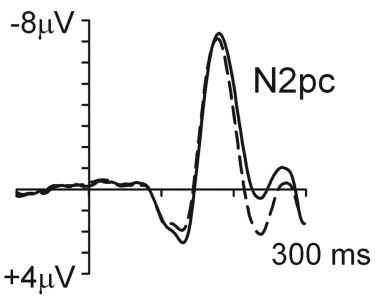

NTCS

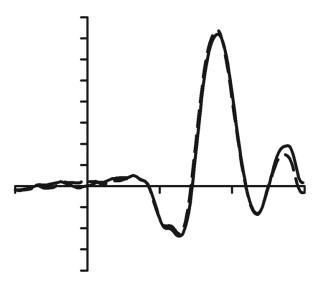

Contralateral
Difference waves

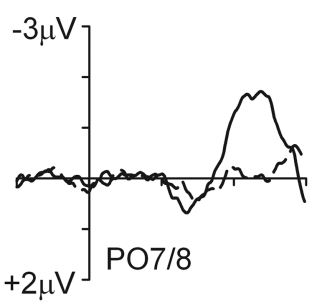

TCS
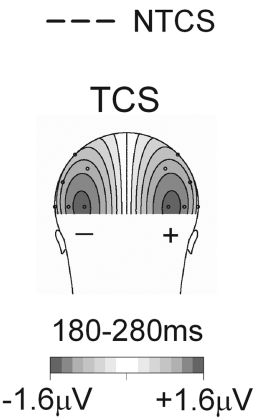

Figure 5. Left panels: Event-related brain potentials (ERPs) elicited in Experiment 2 in the 300-ms interval after cue onset in the target-color singleton (TCS) and nontarget-color singleton (NTCS) cue conditions at posterior electrode sites PO7/8 contralateral (solid lines) and ipsilateral (dashed lines) to the visual hemifield where the color cue was presented. Top right panel: Difference waveforms obtained by subtracting ipsilateral from contralateral ERPs for both singleton cue conditions. Bottom right panel: Topographical map (back view) of N2pc scalp distribution (180- to 280-ms postcue) for TCS cue arrays, constructed by spherical spline interpolation after mirroring the ipsilateral-contralateral difference waveform.

Analogous to Experiment 1, a substantial N2pc was elicited by TCS cues, but not by NTCS cues. This was confirmed by statistical analyses of mean amplitudes in the 180- to 280-ms time window that produced a main effect of contralaterality, $F(1,11)=12.9$, $p<.01, \eta_{\mathrm{p}}^{2}=.54$, and a Cue condition $\times$ Contralaterality interaction, $F(1,11)=16.6, p<.01, \eta_{\mathrm{p}}^{2}=.602$. Separate ANOVAs conducted for each cue condition showed a main effect of contralaterality for the TCS cue, $F(1,11)=15.2, p<.01$, $\eta_{\mathrm{p}}^{2}=.58$, but no contralaterality effect for the NTCS cue, $F(1,11)=1.7, p=.22$.

Frontal N2 in response to cue arrays. Figure 4 (right) shows nonlateralized ERP waveforms obtained in Experiment 2 at anterior electrode sites in response to TCS cue arrays (solid lines) and NTCS cue arrays (dashed lines), together with a scalp distribution map of the ERP difference between these two conditions in the 170- to 270-ms postcue interval. As in Experiment 1, an enhanced frontal negativity was again found for nontarget-color relative to TCS cues. An ANOVA of ERP mean amplitudes in the 170- to 270-ms postcue time window obtained at midline electrodes Fpz, $\mathrm{Fz}$, and $\mathrm{Cz}$ revealed a main effect of cue condition, $F(1,11)=6.2$, $p<.05, \eta_{\mathrm{p}}^{2}=.359$. A significant effect of cue condition was also present at lateral frontal electrodes $\mathrm{F} 3 / 4$ and $\mathrm{F} 7 / 8, F(1,11)=9.1$, $p<.05, \eta_{\mathrm{p}}^{2}=.454$, and the Cue condition $\times$ Hemisphere interaction was again not significant, $F(1,11)=1.7, p=.219$.

$N 2 p c$ component in response to target arrays. Figure 6 (top and middle panels) shows ERPs obtained at lateral posterior elec- trodes PO7/8 ipsilateral and contralateral to the visual field where the target was presented, separately for both cue conditions. All waveforms show the 500-ms interval following cue onset, and thus include both cue- and target-elicited activity. They are shown separately for trials where cues and targets were presented at the same location, for trials where they appeared at different locations in the same hemifield, and for trials where they were presented in opposite hemifields. Because waveforms are now plotted relative to the location of the target, the N2pc triggered by target-color cues (Figure 6, middle panel) is reflected by a contralaterally enhanced negativity on samehemifield trials, but shows up as an ipsilaterally enhanced negativity in the 180- to 280 -ms postcue time window on opposite-hemifield trials. No such early lateralized effects were elicited by nontargetcolor cues (top panel), reflecting the absence of the N2pc to these cues. An N2pc to targets is visible in all waveforms, starting approximately $180 \mathrm{~ms}$ after target onset (i.e., $380 \mathrm{~ms}$ after cue onset).

Figure 6 (bottom panel) shows difference waveforms computed by subtracting ipsilateral from contralateral ERPs for the nontarget-color cue condition, separately for the same-location trials, different-location/same-hemifield trials, and oppositehemifield trials. These difference waves suggest that the onset latency of the target-elicited N2pc following nontarget-color cues differed as a function of cue location: The N2pc appears to start earlier on opposite-hemifield trials than on same-hemifield trials, and to be maximally delayed on trials where cues and targets appeared at the same location. This was confirmed by statistical analyses of target-elicited N2pc latencies for the NTCS cue condition. The overall ANOVA showed a main effect of cue-target position on N2pc latency, $F_{\mathrm{c}}(2,22)=8.3, p<.01, \eta_{\mathrm{p}}^{2}=.429$. The $\mathrm{N} 2 \mathrm{pc}$ emerged earlier on opposite-hemifield trials (160 ms after target onset) than on different-location/same-hemifield trials (186 $\mathrm{ms})$ or same-location trials $(198 \mathrm{~ms})$, and both these differences were significant, $F_{\mathrm{c}}(1,11)=6.3$ and $10.0, p<.03$ and $.01, \eta_{\mathrm{p}}^{2}=$ .363 and .477 , respectively. Importantly, when only samehemifield trials were considered, the N2pc delay for same-location as compared to different-location trials was also significant, $F_{\mathrm{c}}(1,11)=6.6, p<.03, \eta_{\mathrm{p}}^{2}=.373 .^{3}$

As can be seen in Figure 6 (middle panel), an analogous comparison of target-elicited N2pc onset latencies for the TCS cue condition is complicated by the earlier presence of the cue-elicited $\mathrm{N} 2 \mathrm{pc}$ and a subsequent lateralized positivity (see also Lien et al.,

\footnotetext{
${ }^{3}$ An analysis of RTs to targets for these three trial types partially confirmed these $\mathrm{N} 2 \mathrm{pc}$ results. RTs were reliably delayed on same-location trials $(514 \mathrm{~ms})$ relative to opposite-hemifield trials $(506 \mathrm{~ms} ; t(11)=2.3$, $p<.04)$, and the difference between RTs on same-location trials and on different-location/same-hemifield trials $(505 \mathrm{~ms})$ approached significance, $t(11)=1.9, p=.085$. In contrast, there was no significant RTs difference between opposite-hemifield and different-location/same-hemifield trials.

An analogous analysis of target-induced N2pc latencies in the nontargetcolor cue condition of Experiment 1 (not shown in Figures) revealed that an earlier N2pc was elicited by targets on opposite-hemifield trials (152 ms after target onset) relative to same-hemifield/different location trials $(183 \mathrm{~ms})$ and same-hemifield/same-location trials $\left(185 \mathrm{~ms} ; F_{\mathrm{c}}(1,11)=27.5\right.$ and 35.7 , both $p<.001$, both $\left.\eta_{\mathrm{p}}^{2}>.714\right)$, but no reliable onset difference of target N2pc components between same-location and different-location trials, $F_{\mathrm{c}}<1$. However, this difference in target N2pc onset latencies between oppositehemifield and same-hemifield trials in Experiment 1 was not accompanied by significant RT differences between these two types of trials.
} 
NTCS cue
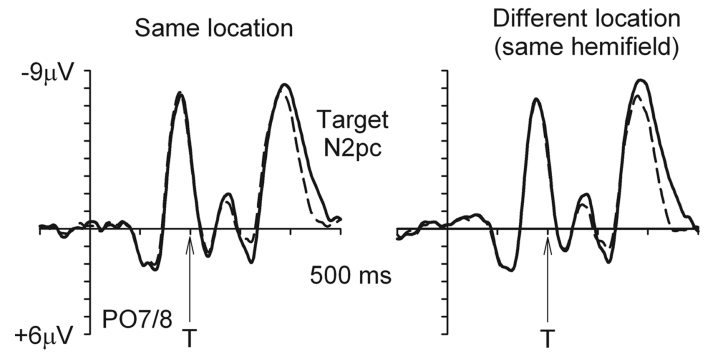

Different location

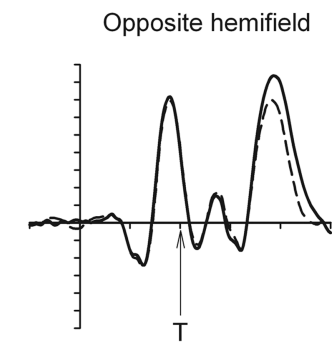

TCS cue

Same location

Different location (same hemifield)

Opposite hemifield
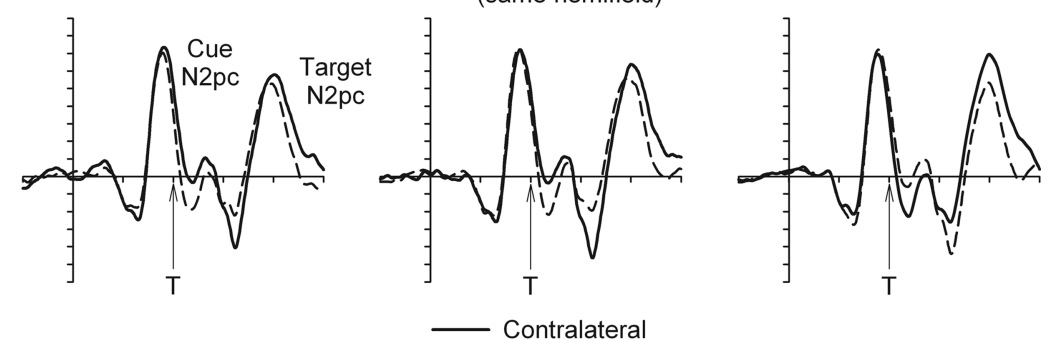

--- Ipsilateral

Difference waves NTCS cue

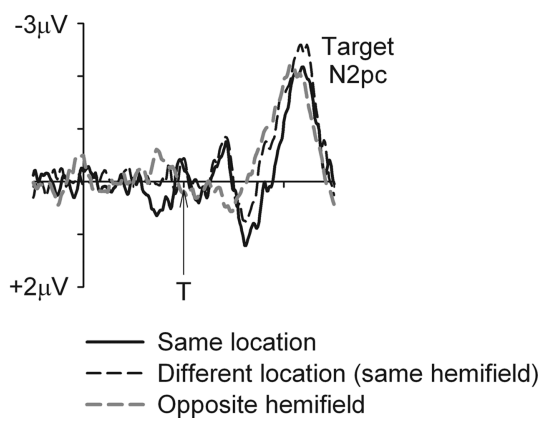

Figure 6. Top and middle panels: Event-related brain potentials (ERPs) obtained in Experiment 2 in the 500-ms interval after cue onset in the nontarget-color singleton (NTCS) and target-color singleton (TCS) cue conditions at posterior electrodes PO7/8 contralateral (solid lines) and ipsilateral (dashed lines) to the visual hemifield where the target stimulus was presented, shown separately for trials where cues and targets were presented at the same location, at different locations in the same hemifield, and in opposite hemifields. ERPs show a cue-elicited N2pc in the TCS cue condition only (with opposite polarity on same-hemifield and different-hemifield trials), and a target-elicited N2pc in both cue conditions. Bottom panel: Difference waveforms obtained by subtracting ipsilateral from contralateral ERPs for the NTCS cue condition, shown separately for same-location trials (solid line), different-location same-hemifield trials (black dashed line), and opposite-hemifield trials (grey dashed line). All plots are time locked to cue onset, and "T" marks the point in time where the target search array was presented.

2008), which are of opposite polarity in same-hemifield and opposite-hemifield trials. A comparison of target-elicited N2pc onset latencies for same-location and different-location/samehemifield trials in the TCS cue condition yielded no reliable difference (205 ms vs. $\left.201 \mathrm{~ms} ; F_{\mathrm{c}}<1\right)$.

\section{Discussion}

The findings of Experiment 2 provide further new evidence that inhibitory mechanisms are elicited in response to percep- tually salient singleton items that do not match a currently active task set. Reducing the SOA between cue and target arrays by $50 \mathrm{~ms}$ relative to Experiment 1 resulted in a small but reliable inverted cueing effect on RTs in the NTCS cue condition, in line with previous observations by Lamy et al. (2004). If this inverted cueing effect was related to location-specific inhibition triggered by nontarget singleton cues, this inhibition could modulate the attentional selection of subsequent targets in a spatially specific fashion, which should be reflected in systematic effects of cue location on the onset latency of N2pc 
components triggered by these targets. This prediction was confirmed in Experiment 2. The target N2pc emerged later on trials where nontarget-color cues and targets appeared at the same location, relative to trials where they appeared at different locations in the same hemifield, or in opposite hemifields (see Figure 6, top panel). This pattern of results strongly suggests that the attentional selection of targets was delayed when they occupied the same position as a preceding task-irrelevant singleton cue, and thus provides new electrophysiological evidence for location-specific inhibition. The observation that target $\mathrm{N} 2 \mathrm{pc}$ components emerged earlier on trials where cues and targets were presented in opposite hemifields than on trials where they appeared on the same side, but at different locations, may point to a spatial gradient in top-down inhibition, with inhibition affecting locations in the same hemifield as a previous task-irrelevant salient event more than locations in the opposite hemifield. However, given that this N2pc latency difference was not reflected by corresponding RT differences between opposite-hemifield trials and different-location/samehemifield trials, this hypothesis requires further corroboration in future experiments.

Experiment 2 also confirmed the new finding of Experiment 1 that task-irrelevant color singleton cues trigger an anterior N2 component. As shown in Figure 4, the overall amplitude and topography of this component was similar across both experiments. Figure 4 suggests that the N2 to NTCS cues emerged somewhat earlier in Experiment 2 relative to Experiment 1. This may be related to the fact that the cue-target SOA was shorter in Experiment 2, which is likely to have increased the temporal demands on top-down inhibition.

Finally, the pattern of N2pc results observed in Experiment 2 in response to TCS and NTCS cues also confirmed the observations from Experiment 1: An N2pc was elicited only for target-color cues, but not for nontarget-color cues. This observation further confirms the conclusion that attentional capture is determined by feature-specific top-down task sets, and is not triggered in a bottom-up fashion by salient visual stimuli irrespective of their task relevance.

\section{General Discussion}

Previous behavioral and ERP studies have provided evidence that attentional capture by salient visual events is determined by top-down task sets (e.g., Eimer \& Kiss, 2008; Folk et al., 1992; Lamy et al., 2004; Lien et al., 2008). This was confirmed in the two experiments reported here. Spatially uninformative targetcolor cues captured attention, as demonstrated by behavioral cueing effects and the presence of reliable N2pc components. In contrast, no behavioral cueing effects and no N2pc components were observed in response to nontarget-color singleton cues, which strongly suggests that salient singleton stimuli do not capture attention when their features are task irrelevant. However, one could still argue that these cues did trigger a rapid transient shift of attention (as postulated by Theeuwes et al., 2000), but that this was not reflected by an early N2pc. Given its latency, the $\mathrm{N} 2 \mathrm{pc}$ is unlikely to be generated during the initial feed-forward analysis of visual information, and has instead been linked to a subsequent phase of spatially selective visual processing that is initiated by re-entrant signals from posterior parietal areas involved in attentional control (e.g., Luck \& Hillyard, 1994b). If the N2pc follows the first parallel stage of visual processing, it may not directly reflect effects of bottom-up salience that are exclusively restricted to this first stage. Although the existence of such salience-induced spatially selective effects on early feed-forward visual processing cannot be ruled out with certainty, previous studies have repeatedly demonstrated that the $\mathrm{N} 2 \mathrm{pc}$ is highly sensitive to the rapid attentional selection of candidate target events (e.g., Eimer \& Kiss, 2007; Hickey et al., 2006; Kiss et al., 2007, 2008a, 2008b; Lien et al., 2008). Therefore, the fact that the presence or absence of an N2pc to color singleton cues was found to be determined by top-down task set is not in line with the "transient capture followed by disengagement" account (see also Folk \& Remington, 2006, for a critical evaluation of behavioral evidence for this hypothesis).

It should be noted that even though most recent ERP studies (e.g., Eimer \& Kiss, 2008; Kiss et al., 2008a; Lien et al., 2008) strongly support the view that top-down task-sets play a dominant role in determining attentional capture, other recent results (Hickey et al., 2006) have suggested that capture is at least partially determined by bottom-up salience. In this study, in which target shape singletons and more salient nontarget color singletons were presented simultaneously in opposite hemifields, a small but reliable N2pc was triggered by color singletons before the $\mathrm{N} 2 \mathrm{pc}$ to shape targets, indicative of rapid attentional capture by task-irrelevant stimuli. These observations suggest that bottom-up factors may sometimes override the impact of top-down task set, especially when there are large salience differences between targets and nontargets. Salience may also affect capture when task sets are less sharply defined, as in the Hickey et al. (2006) study, where two different shape targets changed unpredictably across trials (see also Pinto, Olivers, \& Theeuwes, 2005, for evidence that bottom-up attentional capture is stronger when target identity is uncertain). In the present study, color task sets remained constant throughout an entire experimental session, which may have contributed to the observed dominance of top-down task set over bottom-up salience.

The results discussed so far do not necessarily imply that bottom-up salience does not play any role in attentional capture. To investigate whether the ability of visual events to capture attention in a task-set contingent fashion is affected when their bottom-up salience is reduced, Experiment 1 directly contrasted behavioral and electrophysiological markers of attentional capture in response to target-color cues when their bottom-up salience was either high (in singleton color cue arrays) or low (in heterogeneous color cue arrays). Despite their salience difference, behavioral capture effects and N2pc components for these two types of cues differed only minimally, which suggests that bottom-up salience has a very limited if any role in task-set contingent attentional capture.

Even though large differences in salience had little effect on the capacity of visual events with currently task-relevant features to capture attention, salience appears to be an important factor when stimuli are task irrelevant. Both experiments obtained convergent new electrophysiological evidence that taskirrelevant singletons are subject to location-specific inhibition. An anterior N2 component was observed in response to NTCS 
cue arrays relative to target-color cue arrays (Figure 4), in line with an activation of inhibitory attentional control processes in dorsofrontal brain areas (Folstein \& Van Petten, 2008). Such inhibitory processes might be triggered as a result of the detection of a task-irrelevant feature discontinuity during an initial fast preattentive analysis of the cue stimulus array, to prevent attentional capture by salient stimuli that do not possess taskrelevant attributes. ${ }^{4}$

Further ERP evidence for location-specific inhibition was obtained in Experiment 2. Here, the presence of a small but reliable inverted behavioral cueing effect observed in response to NTCS cues was accompanied by clear effects of previous cue location on the latency of the N2pc to target arrays: The target-elicited N2pc emerged later on trials where nontarget-color cues and targets appeared at the same location than on trials where cues and targets appeared at different locations in the same hemifield, or in opposite hemifields (Figure 6). This location-specific latency shift of the N2pc to target arrays preceded by NTCS cues demonstrates that these cues systematically affected the speed of attentional target selection. Taken together, the ERP modulations observed in the NTCS cue condition suggest that perceptually salient visual events that do not match the current task set trigger inhibitory control mechanisms in frontal areas involved in top-down cognitive control (as reflected by the anterior N2 component), and that the inhibition of such events delays the attentional selection of subsequent targets in a location-specific fashion (as indicated by the latency shift of the target N2pc on same-location trials).

Although the ERP results observed for NTCS cues is consistent with an interpretation in terms of location-specific top-down inhibition of capture by irrelevant singletons, an alternative account should be considered. Folk and Remington (1998, Exp. 3) found that relative to a baseline condition without singleton cues, RTs were delayed on trials where NTCS cues were presented, even though these cues did not produce any spatial cueing effects indicative of attentional capture. They attributed this RT delay to a general distraction effect ("filtering cost") that is driven by the bottom-up salience of color singleton cues, and argued that such filtering costs need to be distinguished from the capture of attention. It is unlikely that the anterior N2 component observed for NTCS is linked to a filtering process as postulated by Folk and Remington (1998). This N2 modulation was quantified as the difference between ERPs to TCS and NTCS cue arrays, which were identical in terms of their salience and only differed with respect to their task-relevance. If filtering costs are exclusively determined by bottom-up salience, and are independent of task set, as assumed by Folk and Remington (1998), they should have been equally present in both cue conditions, and no ERP differences attributable to filtering should have been observed between these conditions. Furthermore, because filtering costs are by their very nature spatially unspecific, they cannot account for the spatially specific effects of cue location on the latency of N2pc components triggered in response to subsequent target arrays.

Overall, the electrophysiological results obtained in the present study for NTCS cues provide new support for the assumption that salient but task-irrelevant stimuli are subject to a rapid and transient top-down inhibition process. Further research is needed to investigate the time course and functional properties of these inhibitory mechanisms in more detail. A confirmation that inhibitory control is critically involved in task-set contingent attentional capture would be important for our understanding of the interactions between endogenous and exogenous attention. Establishing a link between bottom-up salience and inhibition would also demonstrate that the absence of attentional capture by task-set irrelevant singleton stimuli (as demonstrated by behavioral and ERP measures) does not imply that salience is entirely irrelevant for top-down attentional control.

In summary, the present study has provided new insights into the role of bottom-up salience for task-set contingent attentional capture. For visual stimuli with features that match the current task set, variations in their salience have little impact on their capacity to attract attention, demonstrating that capture is primarily mediated by task set, with only a minimal role for salience. For stimuli that do not have task-relevant features, perceptual salience appears to be linked to a transient and location-specific mechanism of top-down inhibition, which may act to prevent attentional capture, and also affects the attentional selection of subsequent target events.

\footnotetext{
${ }^{4}$ Previous ERP studies that have used spatial cueing paradigms have observed a different anterior ERP component that is also related to topdown attentional control: The anterior directing attention negativity (ADAN) is typically elicited $350 \mathrm{~ms}$ after the onset of a central symbolic cue that indicates the direction (left vs. right) of an endogenous shift of spatial attention (e.g., Eimer, Van Velzen, \& Driver, 2002). In contrast to the nonlateralized anterior $\mathrm{N} 2$ component observed in the present study, the ADAN is a lateralized component that is characterized by an enhanced negativity at frontocentral electrodes contralateral to the side of a cued attention shift. While the anterior N2 is suggested to reflect spatially selective top-down inhibition, the ADAN is usually interpreted as reflecting the activity of frontal mechanisms involved in the initiation and control of preparatory spatial orienting.
}

\section{References}

Bacon, W. F., \& Egeth, H. E. (1994). Overriding stimulus-driven attentional capture. Perception and Psychophysics, 55, 485-496.

Brisson, B., \& Jolicœur, P. (2007). Electrophysiological evidence of central interference in the control of visual-spatial attention. Psychonomic Bulletin and Review, 14, 126-132.

Coles, M. G. H., Gratton, G., Bashore, T. R., Eriksen, C. W., \& Donchin, E. (1985). A psychophysiological investigation of the continuous flow model of human information processing. Journal of Experimental Psychology: Human Perception and Performance, 11, 529-553.

Eimer, M. (1993). Effects of attention and stimulus probability on ERPs in a go/no-go task. Biological Psychology, 35, 123-138.

Eimer, M. (1996). The N2pc component as an indicator of attentional selectivity. Electroencephalography and Clinical Neurophysiology, 99, 225-234.

Eimer, M., \& Kiss, M. (2008). Involuntary attentional capture is determined by task set: Evidence from event-related brain potentials. Journal of Cognitive Neuroscience, 20, 1423-1433.

Eimer, M., Van Velzen, J., \& Driver, J. (2002). Crossmodal interactions between audition, touch and vision in endogenous spatial attention: ERP evidence on preparatory states and sensory modulations. Journal of Cognitive Neuroscience, 14, 254-271.

Folk, C. L., \& Remington, R. W. (1998). Selectivity in distraction by irrelevant featural singletons: Evidence for two forms of attentional capture. Journal of Experimental Psychology: Human Perception and Performance, 24, 847-858.

Folk, C. L., \& Remington, R. W. (2006). Top-down modulation of preat- 
tentive processing: Testing the recovery account of contingent capture. Visual Cognition, 14, 445-465.

Folk, C. L., Remington, R. W., \& Johnston, J. C. (1992). Involuntary covert orienting is contingent on attentional control settings. Journal of Experimental Psychology: Human Perception and Performance, 18, $1030-1044$.

Folk, C. L., Remington, R. W., \& Wright, J. H. (1994). The structure of attentional control: Contingent attentional capture by apparent motion, abrupt onset, and color. Journal of Experimental Psychology: Human Perception and Performance, 20, 317-329.

Folstein, J. R., \& Van Petten, C. (2008). Influence of cognitive control and mismatch on the N2 component of the ERP: A review. Psychophysiology, 45, 152-170.

Girelli, M., \& Luck, S. J. (1997). Are the same attentional mechanisms used to detect visual search targets defined by color, orientation, and motion? Journal of Cognitive Neuroscience, 9, 238-253.

Hickey, C., McDonald, J. J., \& Theeuwes, J. (2006). Electrophysiological evidence of the capture of visual attention. Journal of Cognitive Neuroscience, 18, 604-613.

Jolicœur, P., Sessa, P., Dell'Acqua, R., \& Robitaille, N. (2006). Attentional control and capture in the attentional blink paradigm: Evidence from human electrophysiology. European Journal of Cognitive Psychology, $18,560-578$.

Kiss, M., Goolsby, B. A., Raymond, J. E., Shapiro, K. L., Silvert, L., Nobre, A. C., et al. (2007). Efficient attentional selection predicts distractor devaluation: ERP evidence for a direct link between attention and emotion. Journal of Cognitive Neuroscience, 19, 1316-1322.

Kiss, M., Jolicœur, P., Dell'Acqua, R., \& Eimer, M. (2008a). Attentional capture by visual singletons is mediated by top-down task set: New evidence from the N2pc component. Psychophysiology, 45, 1013-1024.

Kiss, M., Van Velzen, J., \& Eimer, M. (2008b). The N2pc component and its links to attention shifts and spatially selective visual processing. Psychophysiology, 45, 240-249.

Lamy, D., \& Egeth, H. E. (2003). Attentional capture in singleton-detection and feature-search modes. Journal of Experimental Psychology: Human Perception and Performance, 29, 1003-1020.

Lamy, D., Leber, A., \& Egeth, H. E. (2004). Effects of task relevance and stimulus-driven salience in feature-search mode. Journal of Experimental Psychology: Human Perception and Performance, 30, 1019-1031.
Lien, M.-C., Ruthruff, E., Goodin, Z., \& Remington, R. W. (2008). Contingent attentional capture by top-down control settings: Converging evidence from event-related potentials. Journal of Experimental Psychology: Human Perception and Performance, 34, 509-530.

Liotti, M., Woldorff, M. G., Perez, R., \& Mayberg, H. S. (2000). An ERP study of the temporal course of the Stroop color-word interference effect. Neuropsychologia, 38, 701-711.

Luck, S. J., \& Hillyard, S. A. (1994a). Electrophysiological correlates of feature analysis during visual search. Psychophysiology, 31, 291-308.

Luck, S. J., \& Hillyard, S. A. (1994b). Spatial filtering during visual search: Evidence from human electrophysiology. Journal of Experimental Psychology: Human Perception and Performance, 20, 1000-1014.

Mazza, V., Turatto, M., Umiltà, C., \& Eimer, M. (2007). Attentional selection and identification of visual objects are reflected by distinct electrophysiological responses. Experimental Brain Research, 181, 531536

Perrin, F., Pernier, J., Bertrand, O., \& Echallier, J. (1989). Spherical splines for scalp potential and current density mapping. Electroencephalography and Clinical Neurophysiology, 72, 184-187.

Pinto, Y., Olivers, C. A., \& Theeuwes, J. (2005). Target uncertainty does not lead to more distraction by singletons: Intertrial priming does. Perception and Psychophysics, 67, 1354-1361.

Theeuwes, J. (1991). Cross-dimensional perceptual selectivity. Perception and Psychophysics, 50, 184-193.

Theeuwes, J., Atchley, P., \& Kramer, A. F. (2000). On the time course of top-down and bottom-up control of visual attention. In S. Monsell \& J. Driver (Eds.), Attention and performance XVIII (pp. 105-124). Cambridge, MA: MIT Press.

Ulrich, R., \& Miller, J. (2001). Using the jackknife-based scoring method for measuring LRP onset effects in factorial designs. Psychophysiology, $38,816-827$

Woodman, G. F., \& Luck, S. J. (1999). Electrophysiological measurement of rapid shifts of attention during visual search. Nature, 400, 867-869.

Received March 19, 2008

Revision received February 13, 2009

Accepted February 18, 2009

\section{E-Mail Notification of Your Latest Issue Online!}

Would you like to know when the next issue of your favorite APA journal will be available online? This service is now available to you. Sign up at http://notify.apa.org/ and you will be notified by e-mail when issues of interest to you become available! 